\title{
Macroinvertebrados bentônicos como indicadores de qualidade de água na Barragem Santa Bárbara, Pelotas, RS, Brasil
}

\author{
Zoobenthics as indicators of the water quality in the Santa Bárbara Dam, Pelotas, RS, Brazil
}

\author{
Sérgio Renato Noguez Piedras ${ }^{1}$ Alex Bager ${ }^{2}$ Paulo Roberto Rocha Moraes ${ }^{2}$ Loraine André Isoldi $^{2}$ \\ Otoniel Geter Lauz Ferreira ${ }^{3}$ Christiane Heemann ${ }^{4}$
}

\section{RESUMO}

O objetivo deste trabalho foi estudar o impacto de dois afluentes da barragem Santa Bárbara sobre o ambiente da mesma, utilizando a ocorrência de invertebrados bentônicos e características químicas da água. Durante nove meses foram realizadas coletas e análises da água e de invertebrados bentônicos nos afluentes Sanga da Barbuda e Sanga do Passo do Cunha e também no interior da bacia de acumulação da barragem. Os resultados mostram que, embora a Sanga da Barbuda e a Sanga do Passo do Cunha apresentem características químicas abaixo do recomendável, no limite aceitável pela legislação que determina os padrões de qualidade da água para abastecimento público, estes afluentes estão, ainda, sendo diluídos de forma satisfatória na bacia de acumulação da barragem. O estudo das relações entre variáveis químicas e invertebrados bentônicos mostra haver correlação significativa entre os baixos níveis de oxigênio dissolvido e a presença de Oligochaeta, sendo que o aumento do número de Oligochaeta indica uma situação de anoxia na barragem Santa Bárbara.

Palavras-chave: zoobentos, saneamento ambiental, gestão hídrica.

\section{ABSTRACT}

The objective of this work was to study the impact of two tributaries from the Santa Bárbara Dam on this environment using the occurrence of zoobenthics and some water chemical characteristics. Monthly gathering and analysis from the water and zoobenthics were done during nine months in the tributaries Sanga da Barbuda and Sanga Passo do Cunha as well as in the inner part of the accumulation basin in the Santa Barbara dam. The results demonstrate that, though

\begin{abstract}
Sanga da Barbuda and Sanga Passo do Cunha present chemical characteristics lower than the recommended but still in the limits acceptable by the law that regulates the water quality standards for the supply, these tributaries are being diluted in a satisfactory way in the accumulation basin in the Santa Barbara dam. The study of the relations between chemical variables and zoobenthics show a significant correlation between low levels of dissolved oxygen and the presence of Oligochaeta; moreover, the increase of Oligochaeta indicates a situation of anoxia in the Santa Barbara Dam.
\end{abstract}

Key words: zoobenthics; environmental sanitation; water administration.

\section{INTRODUÇÃO}

A barragem Santa Bárbara foi construída na década de 1960 pelo Departamento Nacional de Obras e Saneamento (DNOS), com o objetivo de evitar cheias na área urbana de Pelotas, bem como proporcionar reserva para abastecimento público de água potável. Atualmente a Estação de Tratamento de Água do Santa Bárbara, construída à jusante da barragem, trata cerca de 80.000.000 de L.dia ${ }^{-1}$, representando 45\% da água potável consumida pela população do Município de Pelotas.

Com uma bacia de captação de 9.200 hectares, uma superfície de acumulação de 600 hectares e lâmina d'água de 450 hectares, a barragem Santa Bárbara tem como afluentes diversos pequenos arroios,

${ }^{1}$ Escola de Ciências Ambientais, Universidade Católica de Pelotas (UCPel). Rua Professor Araújo, 2081. Pelotas, 96020-360, Pelotas, RS, Brasil. E-mail sergiopiedras@hotmail.com. Autor para correspondência.

${ }^{2}$ Escola de Ciências Ambientais, UCPel, Pelotas, RS, Brasil.

${ }^{3}$ Departamento de Zootecnia, Universidade Federal de Pelotas (UFPel), RS, Brasil.

${ }^{4}$ Escola de Educação, UCPel, RS, Brasil. 
vulgarmente reconhecidos como: Sanga da Granja, a Sanga do Sonho, a Sanga Epaminondas, a Sanga da Gama, a Sanga dos Trilhos, Sanga do Passo do Cunha; Sanga da Barbuda e do próprio arroio Santa Bárbara, que, por sua vez, recebem despejos agrícolas, industriais, domésticos e servem como sistema de drenagem de águas pluviais por onde passam, conduzindo todos estes efluentes à bacia de acumulação da barragem, de forma que existe grande probabilidade deste importante recurso hídrico vir a ter comprometida sua qualidade.

O processo de evolução natural associado ao incremento de matéria orgânica carregada por seus contribuintes traz como conseqüência a alteração do estado natural da água do "lago", ocasionando transtornos ao SANEP (Serviço Autônomo de Saneamento de Pelotas), no tratamento da água para consumo, e à população pelo conseqüente sabor e odor resultante da decomposição de algas cianofíceas.

A eutrofização da barragem Santa Bárbara tem sido discutida e encarada como sendo o problema mais imediato a ser contornado. HUSZAR (1978), estudando o fitoplâncton da barragem, afirma que esta estaria incluída na categoria de lagos eutróficos naturais de RHODE, para o momento estudado, sendo esta classificação baseada na produtividade primária, que, naquele momento, estaria entre 322 e $389 \mathrm{mgC} \mathrm{m}^{-2} \mathrm{dia}^{-1}$. OLIVEIRA (1985) observou que o aumento dos nutrientes, nitratos e fosfatos, na água da barragem, está relacionado ao período de cultivo agrícola no seu entorno, e sugere que o seu estado de eutrofização, deve-se à matéria orgânica que vem sendo acumulada ao longo do tempo.

A barragem sofre um processo de assoreamento, eutrofização, desenvolvimento de algas produtoras de toxinas e neurotoxinas, o que efetivamente é constatado sazonalmente na primavera quando ocorre o aumento da temperatura na região e o conseqüente incremento dos processos metabólicos ambientais ASMUS (2001). Para este autor, estas conseqüências são devidas ao aporte externo de efluentes ricos em matéria orgânica, oriundos principalmente da Sanga da Barbuda.

A gestão ou administração desta questão depende da tomada de decisão do poder público responsável pela qualidade do recurso hídrico, sendo que a avaliação do impacto ambiental dos afluentes da barragem servirá de subsídio à tomada de decisão. BUENO et al. (2003) recomendam o conhecimento das relações interespecificas locais, como passo fundamental para a utilização de macroinvertebrados bentônicos com indicadores de qualidade de água.

A diferenciação dos efeitos resultantes de processos de poluição, e processos modificadores, ao longo da evolução química, biológica, e todos os seus processos indutores que podem reprimir ou favorecer certas formas de vida de modo que esta dinâmica, característica dos sistemas vivos dificulta a diferenciação entre os processos naturais de eliminação de espécies, ou transformação de comunidades, e efeitos semelhantes induzidos pela poluição SCHAFFER(1984).

O uso de bioindicadores tem sido corrente na avaliação de impactos ambientais provocados pela má administração do ambiente, pois animais, plantas, microrganismos e suas complexas interações com o meio ambiente respondem de maneira diferenciada às modificações da paisagem, produzindo informações, que não só indicam a presença de poluentes, mas como estes interagem com a natureza, proporcionando uma melhor indicação de seus impactos na qualidade dos ecossistemas (SOUZA, 2001).

As alterações na qualidade de água, resultantes dos processos de evolução natural e de ação antrópica, se manifestam pela redução acentuada da biodiversidade aquática, em função da desestruturação do ambiente físico, químico e alterações na dinâmica e estrutura das comunidades biológicas, sendo que o uso de bioindicadores (espécies, grupos de espécies ou comunidades) permite uma avaliação integrada dos efeitos ecológicos causados por múltiplas fontes de poluição (CALLISTO et al., 2001). Além disso, os bioindicadores complementam as informações sobre qualidade das águas, especialmente para a avaliação de impactos ambientais decorrentes de descargas pontuais de esgotos domésticos e efluentes industriais.

Os organismos aquáticos, principalmente invertebrados, são os que melhor respondem às mudanças das condições ambientais. Ambientes fortemente impactados mostram poucas espécies que, se estiverem bem adaptadas, podem exibir ótimo desenvolvimento e o monitoramento de estações a montante e a jusante da fonte poluidora, pode identificar as conseqüências ambientais para a qualidade de água e saúde do ecossistema aquático (MATSUMARA-TUNDISI, 1999).

O conhecimento dos organismos aquáticos de um reservatório é de fundamental importância, pois a presença ou ausência de certas espécies serve como indicador do "status" a longo prazo da qualidade de água, além de favorecer, através da manipulação da cadeia alimentar, a melhora da qualidade da água (STRASKRABA \& TUNDISI, 2000).

Neste contexto, foi objetivo deste trabalho estudar as relações entre parâmetros físicos e químicos de água e as comunidades de invertebrados bentônicos, em dois afluentes e na bacia de acumulação 
da barragem Santa Bárbara, de forma a avaliar impacto ambiental destes afluentes.

\section{MATERIAL E MÉTODOS}

O estudo foi desenvolvido no período de março a novembro de 2003. As coletas foram realizadas em dois pontos da bacia de captação da barragem Santa Bárbara, a saber: Na Sanga da Barbuda e na Sanga Passo do Cunha, ambos a 30 metros do seu deságüe na barragem, e um ponto no interior da bacia de acumulação, próximo a taipa, a 50 metros da margem direita da barragem. Em cada um destes pontos, foram tomadas três amostras de bentos e de água. Os pontos foram escolhidos, a partir de análises preliminares nos afluentes da barragem, que caracterizaram variações nos padrões de qualidade da água.

A Sanga da Barbuda, cujas nascentes estão localizadas no entroncamento da BR-116 e a Avenida Fernando Osório, no acesso norte da Cidade de Pelotas, recebe efluentes domésticos a partir do loteamento Sítio Floresta, da Vila Jacob Brod e da Vila da Páscoa. Recebe também efluentes de indústrias de arroz, conservas, frigoríficos e de pescados. A Sanga Passo do Cunha tem nascentes ao norte da barragem e recebe efluentes, predominantemente agrícolas, originados da pecuária leiteira, fruticultura, avicultura e florestamento.

As características físicas e químicas da água de superfície nos pontos de estudo foram determinadas de acordo com APHA (1998). Para coletas e análises de invertebrados bentônicos, foi utilizado um cano de PVC de 40mm de diâmetro para coletar os organismos, conforme sugerido por OLIVEIRA (1986). O sedimento coletado foi conservado e corado em solução de formol a $4 \%$ com o corante Phloxin a $0,1 \mathrm{~g} \mathrm{~L}^{-1}$ (CETESB, 1978). Em laboratório, foi feita a triagem das amostras através de peneiras com abertura de malhas de 500 micras. Os macroinvertebrados encontrados foram selecionados e contados por taxa, sob lupa binocular com aumento até 40 vezes. A seleção e identificação dos organismos foi com base nas chaves de classificação de NEEDHAM \& NEEDHAM(1978) eAPHA(1998).

Os resultados das análises de água nos pontos estudados foram comparados à resolução $n^{\circ}$ 20 do CONAMA (1986), e a ocorrência de invertebrados bentônicos foi avaliada através do índice de diversidade (H') de Shanonn (ODUM, 1988), que considera a riqueza das espécies em relação ao número total de indivíduos presentes no local.

Para análise de agrupamento das unidades amostrais, utilizou-se o critério de variância mínima (soma de quadrados), com base na distância euclidiana com os dados previamente centralizados e normalizados, dentro de variáveis. A transformação dentro de variáveis fez-se necessária, uma vez que os dados são quantitativos de unidades diferentes. A análise de significância dos grupos formados foi obtida através de autoreamostragem ('bootstrap').

Para ordenação das unidades amostrais, utilizou-se o método de análise de componentes principais (PCA). Na PCA, aplicou-se a matriz de semelhança de correlação entre as variáveis. A análise de significância dos eixos formados foi obtida através de autoreamostragem ('bootstrap').

A análise de variância via testes de aleatorização (PILLAR \& ORLÓCI, 1996) foi realizada utilizando-se por base uma matriz de distâncias euclidianas entre unidades experimentais, com os dados previamente normalizados e centralizados e 1000 iterações. Foi considerada o nível de significância de 0,05 .

Todas as análises foram realizadas com o software MULTIV 2.0.3, com 5\% de significância (PILLAR, 1997; PILLAR, 2001).

\section{RESULTADOS E DISCUSSÃO}

A tabela 1 apresenta as médias dos parâmetros físicos e químicos analisados durante o período estudado. Estes dados indicam que a taipa e a Sanga Passo do Cunha atendem a Resolução no 20 do CONAMA (1986), que estabelece os padrões de qualidade para o uso destinado da água, que, no caso, é o tratamento para abastecimento publico. Já a Sanga da Barbuda, que apresentou uma concentração média de $1,7 \pm 0,72 \mathrm{mg} \mathrm{L}^{-1}$ de oxigênio dissolvido, não atende ao requisito de apresentar um mínimo de $4 \mathrm{mg} \mathrm{L}^{-1}$ de oxigênio dissolvido. Esta situação é resultante do lançamento de efluentes domésticos e cloacal dos 2.360 habitantes (ITEPA, 2003) residentes na região, e dos efluentes industriais nela lançados pelas diversas indústrias (arroz, pescado, frigoríficos e conservas) localizadas a montante da sanga.

A Sanga da Barbuda apresentou, também, uma condutividade média de 309Mhos $\mathrm{cm}^{-1} \mathrm{e}$ alcalinidade média de $120,3 \mathrm{mg} \mathrm{L}^{-1}$ de $\mathrm{CaCO}_{3}$, parâmetros esses excessivamente alterados em relação aos demais pontos estudados. Esse resultado pode ser atribuído a efluentes urbanos sem tratamento, originados dos núcleos residenciais sitio Floresta e ilha da Páscoa e dos sistemas de tratamento de efluentes industriais de plantas a montante da sanga, que utilizam soda cáustica para a neutralização dos seus efluentes.

A qualidade de água no interior da barragem, tendo como referência as análises realizadas na taipa, apresenta algumas características alteradas em relação ao registrado por OLIVEIRA (1985). Esse fato é 
Tabela 1 - Médias e desvio padrão dos parâmetros físicos e químicos avaliados nos locais de estudo durante o período estudado

\begin{tabular}{|c|c|c|c|}
\hline Parâmetro & Taipa & Passo do Cunha & Sanga da Barbuda \\
\hline Temperatura $\left({ }^{\circ} \mathrm{C}\right)$ & $15,8 \pm 1,0$ & $15,6 \pm 0,9$ & $14,5 \pm 0,9$ \\
\hline Oxigênio dissolvido (mg. $\mathrm{L}^{-1}$ ) & $7,01 \pm 0,79$ & $4,4 \pm 0,72$ & $1,7 \pm 0,72$ \\
\hline Saturação de $\mathrm{O}_{2}(\%)$ & $71 \pm 7,9$ & $45 \pm 7,2$ & $17 \pm 7,2$ \\
\hline Gás carbônico (mg de $\mathrm{CO}_{2} \mathrm{~L}^{-1}$ ) & $3,4 \pm 6,8$ & $10,1 \pm 6,2$ & $40,0 \pm 6,2$ \\
\hline $\mathrm{pH}$ & $6,9 \pm 0,5$ & $6,6 \pm 0,4$ & $6,8 \pm 0,6$ \\
\hline Condutividade (Mhos.cm ${ }^{-1}$ ) & $44,6 \pm 58$ & $38,1 \pm 53$ & $309,3 \pm 53$ \\
\hline Alcalinidade (mg de $\mathrm{CaCO}_{3} \cdot \mathrm{L}^{-1}$ ). & $14,8 \pm 12,0$ & $14,1 \pm 10,9$ & $120,3 \pm 10,9$ \\
\hline Nitrogênio amoniacal (mg. $\mathrm{L}^{-1}$ ) & $0,78 \pm 0,55$ & $0,46 \pm 0,86$ & $12,04 \pm 6,2$ \\
\hline Fosfato total (mg. $\left.\mathrm{L}^{-1}\right)$ & $0,55 \pm 0,41$ & $0,43 \pm 0,60$ & $5,52 \pm 2,18$ \\
\hline Sólidos totais $105^{\circ} \mathrm{C}\left(\mathrm{mg} . \mathrm{L}^{-1}\right)$ & $61,2 \pm 43,1$ & $105 \pm 91,0$ & $218 \pm 48,4$ \\
\hline
\end{tabular}

constatado, principalmente, no que se refere ao oxigênio dissolvido que, em 1986, apresentava em média uma concentração superior $8,0 \mathrm{mg} \mathrm{L}^{-1}$ contra os 7,01mg L-1, o que significa que houve um aumento no consumo do oxigênio para a decomposição da matéria orgânica e inorgânica que chega á bacia de acumulação.

Em relação à presença de macroinvertebrados bentônicos, o taxa mais comum foi Oligochaeta, predominando em todos os pontos (Tabela 2). A presença de Oligochaeta submetida à

Tabela 2 - Ocorrência dos principais macroinvertebrados bentônicos em número médio de indivíduos por metro quadrado nos locais de amostragem, durante o período de estudo

\begin{tabular}{|c|c|c|c|}
\hline Organismos & Taipa & Passo do Cunha & S. Barbuda \\
\hline \multicolumn{4}{|l|}{ Oligochaeta } \\
\hline Limnodrilus sp. & 9685 & 13920 & 40062 \\
\hline Aulophurus sp. & 1937 & 870 & 11446 \\
\hline Prístina sp. & 1291 & 2610 & 5724 \\
\hline \multicolumn{4}{|l|}{ Nemathelminto } \\
\hline Dorylaimus sp. & 5211 & 4299 & 9450 \\
\hline Dorylaimidae II & 1302 & 1434 & 5550 \\
\hline \multicolumn{4}{|l|}{ Chironomidae } \\
\hline Ablamesmia sp. & 329 & 256 & 182 \\
\hline Pentaneura sp. & 411 & 320 & 228 \\
\hline Procladius sp. & 493 & 384 & 274 \\
\hline Tanypus sp. & 411 & 320 & 228 \\
\hline Chironomus sp. & 6582 & 5120 & 3656 \\
\hline \multicolumn{4}{|l|}{ Plathyelminto } \\
\hline Dalyellidae & 0 & 4 & 0 \\
\hline \multicolumn{4}{|l|}{ Trichoptera } \\
\hline Laptoceridae & 98 & 6 & 0 \\
\hline \multicolumn{4}{|l|}{ Gastropoda } \\
\hline Physa sp. & 243 & 5 & 0 \\
\hline Biomphalaria sp. & 432 & 4 & 0 \\
\hline Plecoptera & 68 & 0 & 0 \\
\hline Odonata & 12 & 0 & 0 \\
\hline Trichoptera & 18 & 14 & 0 \\
\hline
\end{tabular}

análise de correlação com o oxigênio dissolvido, apresentou efeito significativo $\left(\mathrm{y}=97528 \mathrm{x}^{-1,0806}, \mathrm{R}^{2}=\right.$ 0,98 ), sendo que o aumento do número de Oligochaeta, ocorreu com a redução da concentração de oxigênio dissolvido na água (Figura 1, Tabela 3). Este resultado é semelhante ao registrado por MONTANHOLIMARTINS et al. (2004), que, estudando o córrego Bonito, afluente do rio Formoso no Mato Grosso do Sul, obteve correlação significativa de Oligochaeta, com variáveis ambientais que caracterizam locais impactados com poluição orgânica.

A predominância de Oligochaeta, de acordo com OLIVEIRA (1985), em determinados períodos e locais da barragem Santa Bárbara, deve-se ao aumento da deposição de matéria orgânica originada da decomposição de macrófitos aquáticos, que, no momento, está sendo incrementada através dos afluentes da barragem.

A abundância de espécies diferentes de Oligochaeta aquáticos pode ser uma boa indicação da poluição da água RUPPERT \& BARNES (1996). BUBINAS \& JAMINIENÉ (2001) classificam os Chironomidae e Oligochaeta como os invertebrados bentônicos mais tolerantes a poluição. MATSUMURATUNDISI (1999) afirma que, dentre os bioindicadores, há grupos de espécies diretamente relacionados a um determinado agente poluidor ou a uma forma natural potencialmente poluente. Altas densidades de Oligochaeta e Chironomidae são indicadores de elevados teores de matéria orgânica.

A análise de variância via teste de aleatorização (Figura 2), considerando os dados multivariados, indicou diferenças significativas entre taipa e a Sanga da Barbuda e a Sanga Passo do Cunha e a Sanga da Barbuda. Taipa e a Sanga Passo do Cunha não apresentaram diferenças significativas. Não houve efeito da interação entre local e época de avaliação. Também não houve efeito significativo das épocas 


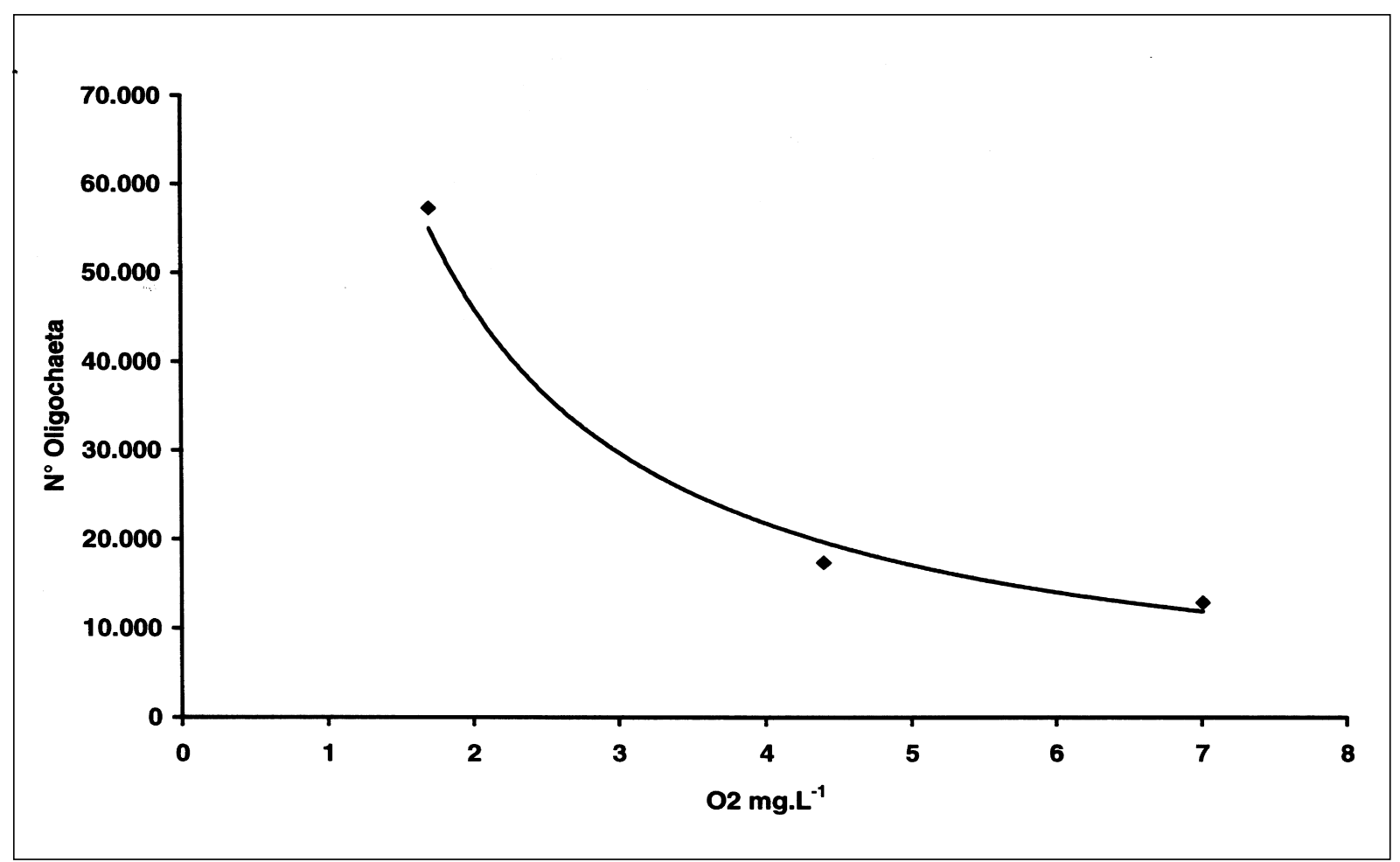

Figura 1 - Correlação entre a concentração de oxigênio dissolvido e o número de Oligochaeta, considerando todos os pontos estudados $\left(y=97528 x^{-1,0806}, \quad R^{2}=0,98\right)$.

isoladamente, provavelmente pelo fato dos dados terem sido analisados de forma conjunta (multivariada).

Os índices de diversidade de $\mathrm{H}^{\prime}=1,75$ na taipa, $H^{\prime}=1,60$ na sanga Passo do Cunha e $H^{\prime}=1,44$ na sanga da Barbuda, são menores que os obtidos por OLIVEIRA (1985), que registrou H’ > 2,0 em vários pontos da barragem. Esta redução da diversidade pode ser atribuída à deterioração da qualidade de água. Estes resultados, se comparados ao trabalho de WILHM \& DORRIS (1968), que sugerem a seguinte regra geral para avaliar a qualidade da água: $\mathrm{H}^{\prime}<1,0$ - forte poluição; H' = 1,0 - 3,0 - poluição moderada e H'> 3,0 - água sem poluição, indicam que todo o sistema apresenta índices de poluição moderada.

Tabela 3 - Correlações das variáveis bióticas e abióticas, com os dois primeiros componentes principais

\begin{tabular}{|c|c|c|c|c|c|}
\hline Variáveis & Eixo 1 & Eixo 2 & Variáveis & Eixo 1 & Eixo 2 \\
\hline $\mathrm{T}^{\circ} \mathrm{C}$ ar & 0,23114 & $-0,83433$ & Oligochaeta & $-0,8645$ & $-0,19447$ \\
\hline $\mathrm{T}^{\circ}$ Cágua & 0,39899 & $-0,71296$ & Chironomidae & 0,30552 & $-0,67496$ \\
\hline $\mathrm{O}_{2}$ & 0,875 & 0,23579 & Nemathelminto & $-0,7305$ & $-0,18906$ \\
\hline $\mathrm{CO}_{2}$ & $-0,9008$ & $-0,02792$ & Plecoptera & 0,20309 & 0,30694 \\
\hline Saturação & 0,90891 & 0,12820 & Ephemeroptera & 0,22333 & $-0,16620$ \\
\hline $\mathrm{pH}$ & 0,36917 & $-0,34566$ & Odonata & 0,28262 & $-0,66856$ \\
\hline Alcalinidade & $-0,87834$ & $-0,29508$ & Plathelminto & 0,19684 & $-0,01306$ \\
\hline \multirow[t]{2}{*}{ Condutividade } & $-0,86671$ & $-0,28166$ & Trichoptera & $-0,09695$ & 0,51291 \\
\hline & & & Gastropoda & $-0,13433$ & 0,49397 \\
\hline
\end{tabular}

Componente principal eixo 1 explicou 33\% da variabilidade total dos dados Componente principal eixo 2 explicou $18 \%$ da variabilidade total dos dados 


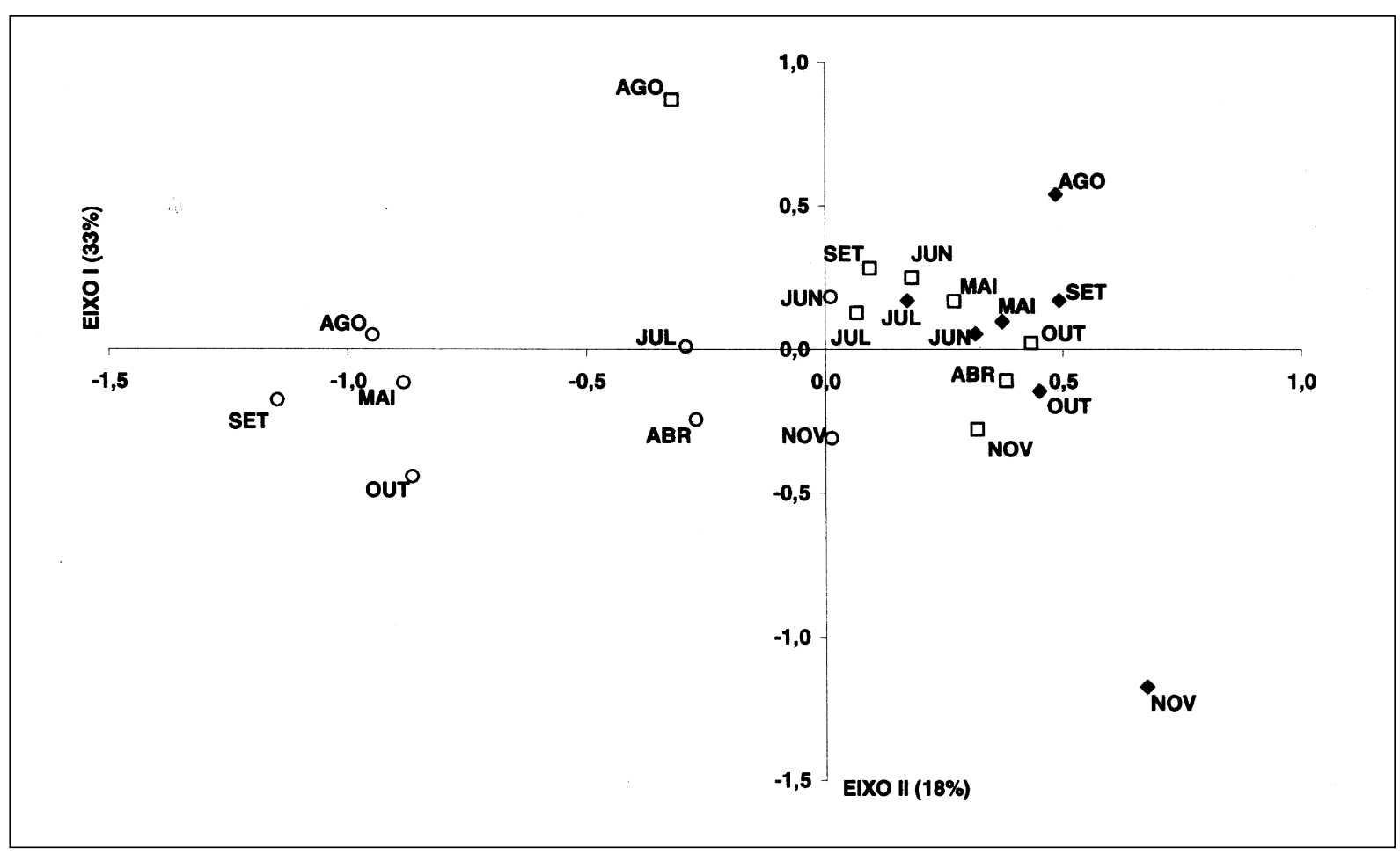

Figura 2- Diagrama de dispersão dos pontos de amostragem e respectiva data, ordenados pelo método de componentes principais, tendo como base uma matriz de correlação entre variáveis. Círculos representam a Sanga da Barbuda, Quadrados representam a Sanga Passo do Cunha e Losangos representam a Taipa.

Estas contradições são explicadas por STRIXINO \& NASCIMENTO (2000), quando afirmam que, embora o princípio do uso de bioindicadores seja global, devem ser desenvolvidos estudos locais, que proporcionem a compreensão das relações bioecológicas de cada região. Esses autores ainda afirmam que esses estudos devem abranger períodos de tempo suficientes para que sejam diminuídas as influências das variações climáticas e econômicas momentâneas, sendo este um dos fatores que podem ter afetado os resultados aqui apresentados. Ainda TUNDISI (1999) afirma que o desenvolvimento de teorias para compreender e analisar a complexidade dos reservatórios é fundamental para acompanhar as mudanças periódicas e planejar usos múltiplos.

O desenvolvimento de programas de biomonitoramento depende da produção de informações resultantes de pesquisas científicas sobre padrões e avaliações dos riscos de ocorrência de impactos ambientais no ecossistema de interesse. Esses dados, quando disponibilizados às agências governamentais de controle ambiental, permitirão o estabelecimento de padrões de classificação da qualidade da água e seus respectivos usos (CALLISTO et al., 2001).

\section{CONCLUSÕES}

A barragem Santa Bárbara, próximo à taipa, apresenta águas com características químicas e biológicas satisfatórias para tratamento e abastecimento público. Já Sanga da Barbuda, que recebe quantidades excessivas de resíduos domésticos e industriais oferece sério risco ambiental para a barragem, e a Sanga Passo do Cunha apresenta características físicas e químicas da água comprometidas, mas, no limite mínimo aceitável, segundo a legislação, para abastecimento público, de forma que medidas mitigadoras deve ser tomadas.

Os Oligochaeta são bioindicadores de qualidade de água na barragem Santa Bárbara, sendo que o aumento do seu número indica situação de anoxia.

\section{REFERÊNCIAS}

APHA. Standard methods for the examination of water and wastewater. New York: American Public Health Association, 1998. 824p.

ASMUS, A.E. Projeto Arroio Santa Bárbara: problemática ambiental, social e econômica. Projeto NEPA. Pelotas: UCPel, 2001. 5p.

Ciência Rural, v.36, n.2, mar-abr, 2006. 
BUBINAS, A.; JAGMINIENÉ, I. Bioindication of ecotoxity according to community structure of macrozoobenthic fauna. Acta Zoológica Lituanica, Vilnius, v.11, n.1, p. 90-99, 2001.

BUENO, A.A.P. et al. Estrutura da comunidade de invertebrados bentônicos em dois cursos d'água no Rio Grande do Sul, Brasil. Revista Brasileira de Zoologia, São Paulo, v.1, n.20 p.115125, 2003.

CALLISTO, M. et al. Macroinvertebrados bentônicos como ferramenta para avaliar a saúde dos riachos. Revista Brasileira de Recursos Hídricos, Florianópolis, v.1, n.6, p.71-82, 2001.

CETESB. Determinações biológicas. Normatização técnica. NT-06. São Paulo, 1978. 276p.

CONAMA. Resolução n. 20, de 18 de junho de 1986. Brasília. Diário Oficial da União - 30/07/1986. 20p.

HUSZAR, V.M.L. Estudo quali-quantitativo e produtividade do fitoplâncton do lago da Barragem Santa Bárbara: UCPel. Pelotas: UCPel, 1978. 4p. (Documento Interno Ipemafla).

ITEPA. Banco de Dados da Zona Sul - RS. Pelotas, 2003. CD-Rom. (Boletim n. 14).

MATSUMURA-TUNDISI, T. Diversidade de zooplancton em represas do Brasil. In: HENRY, R. Ecologia de reservatórios. São Paulo: FAPESP/FUNDIBIO, 1999. p.41-54.

MONTANHOLI-MARTINS, M.C. et al. Avaliação da qualidade da água do córrego Bonito (Bonito/MS) por meio das variáveis bióticas e abióticas. Capturado em 14 mai. 2004. On-line. Disponível na Internet http:// www.pantanal2002.ucdb.br/eixos/eixo03/e3_10pdf.

NEEDHAM, J.G.; NEEDHAM, P.R. Guia para el estúdio de los seres vivos de las águas dulces. Barcelona: Reverte, 1978. 82p.

ODUM, P.E. Ecologia. Rio de Janeiro: Guanabara Koogan, 1988. 434p.

OLIVEIRA, J.E.C. Barragem Santa Bárbara, Pelotas, Rio Grande do Sul - Observações sobre o bentos profundal.
São Paulo. 1985. 135f. Tese (Doutorado em Ciências Zoologia) - Universidade de São Paulo.

PILLAR, V.D.; ORLÓCI, L. On randomization testing in vegetation science: multifactor comparisons of releve groups. Journal of Vegetation Science, Berlim, v.7, p.585-595, 1996.

PILLAR, V.D.P. Multivariate exploratory analysis an randomization testing with MULTIV. Coenoses. Gorizia, Roma, v.12, p.145-148.1997.

PILLAR, V.D.P. MULTIV: aplicativo para análise multivariada e testes de hipóteses - versão 2.0.3. Porto Alegre: Departamento de Ecologia, UFRGS, 2001. CDRom.

SHAFFER, A. Fundamentos de ecologia e biogeografia das águas continentais. Porto Alegre: UFRGS, 1984. 532p.

RUPPERT, E.E. ; BARNES, R. Zoologia dos invertebrados. São Paulo: Roca, 1996. 1125p.

STRASKRABA, M.; TUNDISI, J.G. Gerenciamento da qualidade de águas de represas. In. ______. Diretrizes para o gerenciamento de lagos. São Carlos. ILEC, 2000. 280p.

STRIXINO, S.T.; NASCIMENTO, V.M. Indicadores básicos de qualidade ambiental para bacias hidrográficas: macroinvertebrados bentônicos como indicadores biológicos. In: CURSO TEÓRICO PRÁTICO SOBRE BIOINDICADORES DE QUALIDADE DA ÁGUA, 2000, Jaguariuna. Anais.... Jaguariuna: EMBRAPA, 2000. CD-Rom.

SOUZA, P.A.P. Importância do uso de bioindicadores de qualidade: o caso específico das águas. In: FELICIDADE, N. et al. Uso e gestão dos recursos hídricos no Brasil. São Carlos: Rima, 2001. p.55-66.

TUNDISI, J.G. Reservatórios como sistemas complexos: teoria, aplicação e perspectivas para usos múltiplos. In: HENRY, R. Ecologia de reservatórios. São Paulo: FAPESP/FUNDIBIO, 1999. p.21-38.

WILHM, J.; DORRIS, T. Biological parameters for water quality criteria. Biological Science, Washington, DC, n.18, p.477481, 1968. 